\title{
An optimization research on groove textures of a journal bearing using particle swarm optimization algorithm
}

\author{
Xiangyuan Zhang, Chongpei Liu*, and Bin Zhao \\ College of Power and Energy Engineering, Harbin Engineering University, Harbin, PR China
}

Received: 14 September 2020 / Accepted: 18 December 2020

\begin{abstract}
This study aims to optimize the distributions of groove textures in a journal bearing to reduce its friction coefficient. Firstly, A lubrication model of a groove textured journal bearing is established, and the finite difference and overrelaxation iterative methods are used to numerically solve the model. Then, the friction coefficient is adopted as the fitness function and the groove lengths are optimized by particle swarm optimization (PSO) algorithm to evolve the optimal distributions. Furthermore, the effects of eccentricity ratios and rotary speeds on optimal distributions of groove textures are also discussed. The numerical results show the optimal distributions of groove textures are like trapeziums under different eccentricity ratios and rotary speeds, and the trapeziums become slenderer with increasing of eccentricity ratios. It is also found that the reductions of friction coefficients by optimal groove textures are more significant under lower eccentricity ratios. Briefly, this study may provide guidance on surface texture design to improve the tribological performance of journal bearings.
\end{abstract}

Keywords: Journal bearing / groove textures / friction coefficient / PSO algorithm

\section{Introduction}

As a feasible way to conserve oil and debris on contact surfaces, surface textures have been researched over past decades and widely used in many applications [1], such as vibration cutting tools [2], mechanical seals [3], gas face seals [4], slipper bearing [5], thrust bearing [6], piston rings [7], and medical devices $[8,9]$. These researches showed the applying of surface textures can reduce wear and increase load carrying capacity, which is helpful to prolong service lives of mechanical components, save energy and protect environment.

The journal bearing with surface textures also aroused general concerns of researchers, and the presetting of texture distributions is commonly used in their studies. In order to maximize load carrying capacity and minimize friction torque, Shinde and Pawar [10] adopted Taguchi and grey relational analysis methods to design groove textures with considering groove location, width, gap, height, and numbers. Their optimal solution can increase load carrying capacity by $51.01 \%$ and reduce friction torque by $9.84 \%$. Kango et al. [11] compared the bearing performance between grooves and spherical textures under given eccentricity ratios. Their results showed when compared with spherical textures, grooves can show maximum reductions in bearing performance parameters.

\footnotetext{
* e-mail: ssdpz041@outlook.com
}

Manser et al. [12] showed the bearing performance can be positively affected by partial textures, and the optimal locations are depending on the working conditions and geometry parameters. Yu et al. [13] and Lin et al. [14] showed the load carrying capacity can be increased when textures are in rising part of pressure field, and vice versa. However, some studies have led to conflicting conclusions. Tala-Ighil et al. $[15,16]$ indicated the extra hydrodynamic lift in bearing can be generated when textures are in declining part of pressure field. Shinde and Pawar [17] showed among three partial grooving distributions $\left(90^{\circ}-180^{\circ}, 90^{\circ}-270^{\circ}, 90^{\circ}-360^{\circ}\right)$, the first distribution can maximize pressure increase and the last distribution can minimize friction loss. Their study indicated the optimal grooving distribution may depend on the optimization goal.

The above literatures have made important contributions on texture researches, but the texture distributions are preset in their researches, which probably miss the global optimal design. To resolve this issue, some optimization algorithms are adopted by researchers, such as GA (genetic algorithm) [18-20] and neural network [21]. To design an optimal bushing profile for a journal bearing, Pang et al. [18] conducted a multi-objectives optimization (minimum friction power loss and minimum leakage flowrate) by NSGA-II (modified non-dominant sort in genetic algorithm). Their results showed the optimal profile can be obtained at the profile of order $n=2$, where $n$ is the order of Fourier series and can be determined by the 
gradual method for increasing Fourier series orders. To obtain minimum friction coefficients of a journal bearing and bearing slider, Zhang et al. [19,20] adopted GA to optimize the coverage area of circular dimples. In their researches, bearing surface is divided into certain numbers of grids and the dimple in each grid center existing or not is marked with 1 or 0 , respectively. Then GA is used to evolve the solution process and the final optimal coverage area is like a semi-elliptical shape. Sinanoglu et al. [21] experimentally and theoretically researched the influences of shaft surface textures on film pressure and consequently on load by proposed neural network. The shaft surfaces included two types: trapezoidal and saw surfaces. Their results showed the shaft with trapezoidal surface has larger load carrying capacity than the shaft with saw surface.

The above literatures have showed the genetic algorithm and neural network can be successfully employed in surface texture design for journal bearings, while the PSO algorithm may provide a more convenient way for this issue. Particle swarm optimization (PSO), presented by Eberhart and Kennedy $[22,23]$ in 1995, is an optimization method motivated by behaviors of bird flocking/roosting. In view of this algorithm, the individual members establish a social network and can benefit from previous experiences and discoveries of the other members. PSO algorithm is easier to implement because the swarm are updated only by updating the particle velocity and position vectors, which shows this approach has great potentials for use in the designs for air foil bearing [24], rolling element bearing [25] and magnetorheological (MR) bearing [26].

Although PSO has been used in some previous researches, few scholars adopted this algorithm to optimize surface textures for a journal bearing. The novelty of this study is to optimize the distributions of groove textures in a journal bearing to reduce its friction coefficient by PSO algorithm. The effects of eccentricity ratios and rotary speeds on optimal distributions of groove textures are also discussed. Overall, the optimization idea in this study may be helpful for journal bearings to improve their tribological performance.

\section{Description of a journal bearing with groove textures}

Generally, textures located in convergent area is more favorable for improving tribological performance [19]. In this study, the convergent area is covered with groove textures, as illustrated in Figure 1. A detailed description of groove textures is illustrated in Figure 2. The detailed parameters of a journal bearing and groove textures are given in Tables 1 and 2. Note the oil is assumed as isoviscous incompressible fluid.

As Table 2 shows, $\theta_{s}, \theta_{e}, L_{c}, L_{g}$ and $d_{g}$ are fixed, while $L_{a}$ is variable from 0 to $34 \mathrm{~mm}$. Note the groove length $0 \mathrm{~mm}$ means there is no groove and groove length $34 \mathrm{~mm}$ means the longest groove is slightly shorter than bearing width. The groove numbers $N_{g}$ is

$$
N_{g}=\left[\frac{R\left(\theta_{e}-\theta_{s}\right) \times(\pi / 180)}{\left(L_{c}+L_{g}\right)}\right]+1
$$

According to the above geometric parameters, $N_{g}=13$.

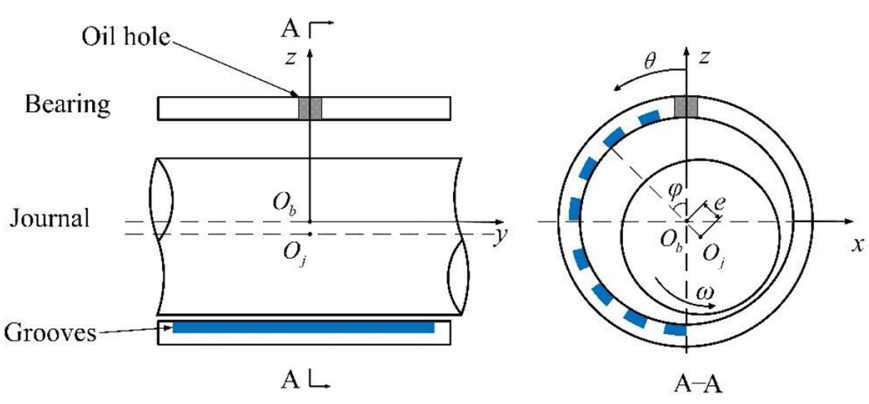

Fig. 1. A textured journal bearing.

\section{Lubrication model}

\subsection{Film thickness}

As illustrated in Figure 1, film thickness $h$ can be obtained by equation (2)

$$
h=c[1+\varepsilon \cos (\theta-\varphi)]+\delta_{t e x}
$$

where $c$ is the radial clearance, $\varepsilon$ the eccentricity ratio $\left(\varepsilon=e / c, e\right.$ eccentricity), $\varphi$ the attitude angle, $\delta_{t e x}$ the clearance added by groove textures.

\subsection{Reynolds equation}

The Reynolds equation under steady operating conditions is shown below [19]

$$
\frac{\partial}{\partial x}\left(\frac{h^{3}}{12 \mu} \cdot \frac{\partial p}{\partial x}\right)+\frac{\partial}{\partial y}\left(\frac{h^{3}}{12 \mu} \cdot \frac{\partial p}{\partial y}\right)=\frac{U}{2} \frac{\partial h}{\partial x}
$$

where $U$ is the relative velocity, $\mu$ the oil viscosity, $p$ the film pressure. For the journal bearing, $U=R \omega, R$ is the journal radius, $\omega$ its angular velocity. The employed Reynolds equation can be obtained by replacing the variable $x$ with $R \theta$ in equation (3), as expressed in equation (4)

$$
\frac{1}{R^{2}} \frac{\partial}{\partial \theta}\left(\frac{h^{3}}{\mu} \cdot \frac{\partial p}{\partial \theta}\right)+\frac{\partial}{\partial y}\left(\frac{h^{3}}{\mu} \cdot \frac{\partial p}{\partial y}\right)=6 \omega \frac{\partial h}{\partial \theta}
$$

\subsection{Load carrying capacity}

Integrate film pressure $p$ over whole the computation domain, then load carrying capacity $F_{b}$ is obtained, as expressed in equations (5) and (6)

$$
\left\{\begin{array}{c}
F_{x}=+\int_{0}^{B} \int_{0}^{2 \pi} p R \sin \theta d \theta d y \\
F_{z}=-\int_{0}^{B} \int_{0}^{2 \pi} p R \cos \theta d \theta d y \\
F_{b}=\sqrt{F_{x}^{2}+F_{z}^{2}}
\end{array}\right.
$$




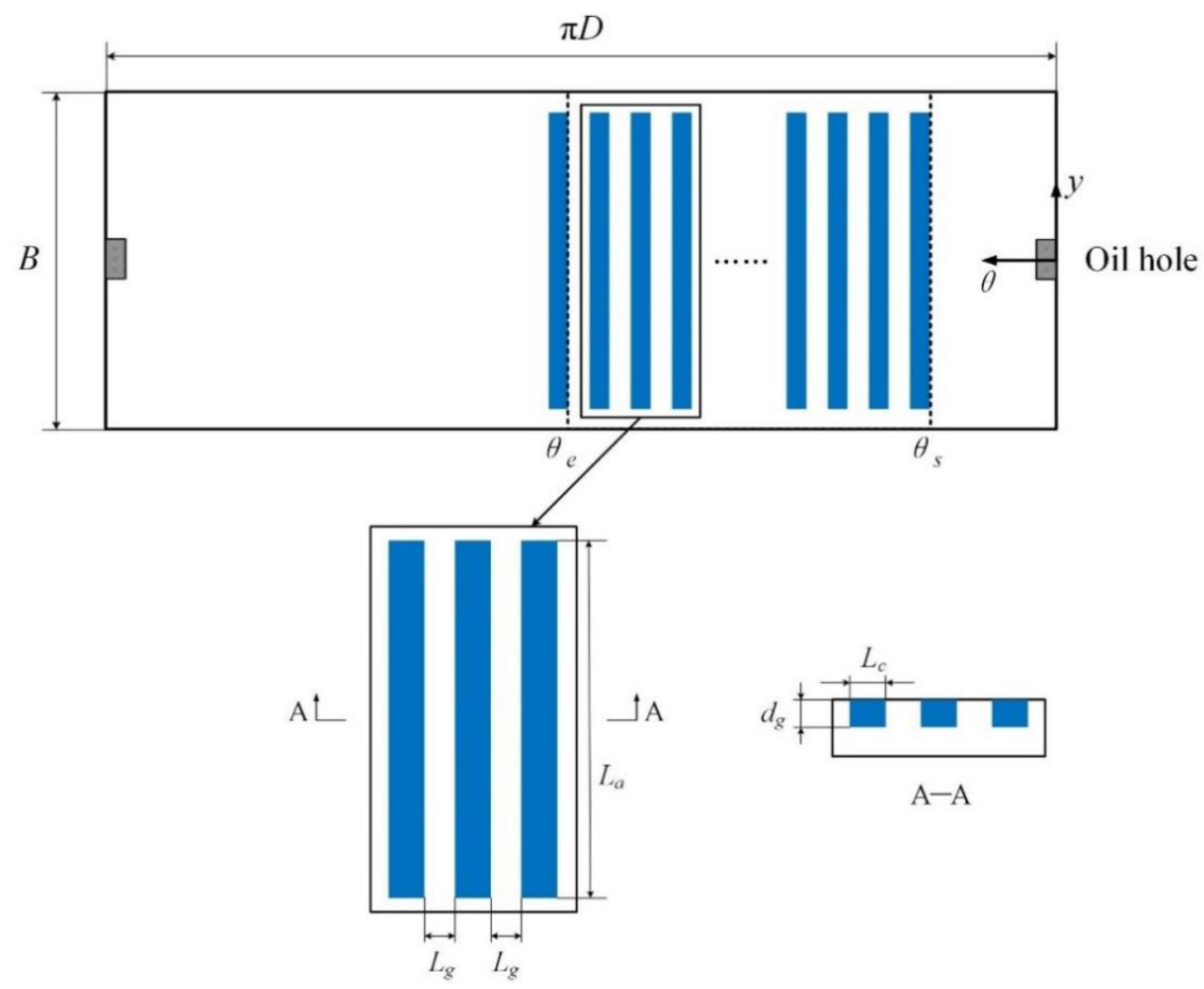

Fig. 2. Groove textures in bearing inner surface.

Table 1. Detailed parameters of a journal bearing.

\begin{tabular}{llll}
\hline Parameters & Values & Parameters & Values \\
\hline Oil viscosity $\mu(\mathrm{Pa} \cdot \mathrm{s})$ & 0.088 & Radial clearance $c(\mathrm{~mm})$ & 0.057 \\
Bearing diameter $D(\mathrm{~mm})$ & 35 & Oil hole diameter $d_{h}(\mathrm{~mm})$ & 5 \\
Bearing width $B(\mathrm{~mm})$ & 35 & Rotary speed $N(\mathrm{rpm})$ & 2000 \\
\hline
\end{tabular}

Table 2. Geometric parameters of groove textures.

\begin{tabular}{lll}
\hline Parameters & Interpretations & Values \\
\hline$\theta_{s}$ & Start position of the first groove $\left(^{\circ}\right)$ & 10 \\
$\theta_{e}$ & Start position of the last groove $\left(^{\circ}\right)$ & 180 \\
$L_{a}$ & Groove length in axial direction $(\mathrm{mm})$ & $0-34$ \\
$L_{c}$ & Groove width in circumferential direction $(\mathrm{mm})$ & 2 \\
$L_{g}$ & Groove gap in circumferential direction $(\mathrm{mm})$ & 2 \\
$d_{g}$ & Groove depth $(\mu \mathrm{m})$ & 10 \\
\hline
\end{tabular}

\subsection{Friction coefficient}

The friction $f_{b}$ arising from the shearing effect of oil can be calculated by equation (7)

$$
f_{b}=\int_{0}^{B} \int_{0}^{2 \pi}\left(\frac{\mu U}{h}+\frac{h}{2 R} \frac{\partial p}{\partial \theta}\right) R d \theta d y
$$

(7) In numerical solution, the bearing inner surface is divided into rectangular grids, and nodes numbers in 
Table 3. Comparisons of Sommerfeld numbers with Pinkus and Sternlicht [27].

\begin{tabular}{llll}
\hline & This study & $\begin{array}{l}\text { Pinkus and Sternlicht }[27] \\
(B / D=1)\end{array}$ & Relative deviation (\%) \\
\hline$\varepsilon=0.1$ & 1.334 & 1.350 & 1.185 \\
$\varepsilon=0.2$ & 0.635 & 0.632 & 0.475 \\
$\varepsilon=0.3$ & 0.391 & 0.382 & 2.356 \\
$\varepsilon=0.4$ & 0.262 & 0.261 & 0.383 \\
$\varepsilon=0.5$ & 0.179 & 0.179 & 0 \\
\hline
\end{tabular}

circumferential and axial directions are $n_{\theta}$ and $n_{y}$, respectively. The equation (4) is discretized by finite difference method, and the pressure is solved by overrelaxation iterative method. The rupture area of oil film is determined by Reynolds boundary conditions, and the pressure in oil hole and both ends of bearing are zero. The pressure convergence criteria $\varepsilon_{p}$ is $10^{-5}$, and the solution process will be terminated if $\left|\Delta P_{i}\right| /\left|P_{i}\right|<\varepsilon_{p}$ is satisfied, then the friction coefficient can be calculated by equations (5)-(8). The programming platform is Intel Xeon E5-2630 at $2.3 \mathrm{GHz}$ with $64 \mathrm{~GB}$ RAM.

It is necessary to verify the model before subsequent analysis. The Sommerfeld number $S$ are calculated based on the researched journal bearing, and the results are compared with Pinkus and Sternlicht [27], as illustrated in Table 3. It can be seen the Sommerfeld numbers calculated by this study agree well with literature's results, which shows good accuracy of this model.

\section{Particle swarm optimization algorithm}

General researches consider the grooves have an equal length for convenience. In the authors' opinion, grooves with unequal lengths may bring more benefits. In this study, groove lengths are optimized by PSO algorithm. Figure 3 shows the computational process of PSO, which includes following steps:

(1) Initialize a particle swarm with 30 particles to be randomly generated. The swarm size $N p=30$ is selected from 20,30, 50 and 70, which can balance the contradiction between computing time and population diversity. The dimension of each particle is $N_{g}$, and the particle elements are random groove lengths.

(2) Calculate the particle fitness according to initial swarm. The friction coefficient $\mu_{f}$ is adopted as the fitness function.

(3) Search the local best pbest and global best gbest.

(4) Perform the iterative calculation. This step is the key of PSO algorithm, which can be subdivided into following substeps:

(4-a) Update the particle velocity vector $v_{i d}$ and position vector $x_{i d}$

$v_{i d}^{T+1}=\omega_{i} v_{i d}^{T}+c_{1} r_{1}\left(\right.$ pbest $\left._{i}^{T}-x_{i d}^{T}\right)+c_{2} r_{2}\left(g b e s t^{T}-x_{i d}^{T}\right)$

$$
x_{i d}^{T+1}=x_{i d}^{T}+v_{i d}^{T+1}
$$

where $i$ is the particle number, $i=1,2,3, \ldots, 30 ; d$ the $N_{g}$ dimensions, $d=1,2,3, \ldots, N_{g} ; \omega_{i}$ the inertia weight. According to Shi and Eberhart [28], PSO has the best chance to find global optimum if $\omega_{i}$ satisfies $0.9 \leq \omega_{i} \leq 1.2$, here $\omega_{i}=0.9 ; c_{1}$ and $c_{2}$ the learning factors, $c_{1}=c_{2}=2$ [28]; $r_{1}$ and $r_{2}$ the random numbers between 0 and 1 [28]; pbesti ${ }^{T}$ the local best; gbest ${ }^{T}$ the global best; $T$ the index of generations; $T_{\max }$ the maximum index of generations.

It is necessary to check if the new velocity and position vectors satisfy boundary conditions:

$$
\begin{gathered}
\left\{\begin{array}{l}
v\left(v>v_{\max }\right)=v_{\max } \\
v\left(v<v_{\text {min }}\right)=v_{\text {min }}
\end{array}\right. \\
\left\{\begin{array}{l}
x\left(x>x_{\max }\right)=x_{\max } \\
x\left(x<x_{\text {min }}\right)=x_{\text {min }}
\end{array}\right.
\end{gathered}
$$

where $v_{\max }=4, v_{\min }=-4$. The velocity range $[-4,4]$ is determined through a multitude of trials, which can help the particles avoid flying past good solution areas or trapping into local optimal solution [29]. $x_{\max }$ and $x_{\min }$ are the maximum and minimum of groove lengths, $x_{\max }=34 \mathrm{~mm}$, and $x_{\min }=0 \mathrm{~mm}$.

(4-b) Update the particle fitness according to new swarm.

(4-c) Update the local best pbest and global best gbest.

(4-d) Exit the loop if $T$ is greater than $T_{\max }=100$; Otherwise, return to (4-a) and repeat (4-a)-(4-d).

(5) Output the final optimal solution: gbest and $\mu f_{\text {best }}$.

\section{Results and discussions}

The mesh refinement is firstly performed based on smooth bearing. The friction coefficients $\mu_{f}$ under $\varepsilon=0.2,0.4, \varphi=0$ of different mesh schemes $n_{\theta} \times n_{y}$ are shown in Figure 4, which shows $\mu_{f}$ tends to stabilize gradually with denser grids. Considering the accuracy and solving speed, $551 \times 177$ mesh is adopted. In this scheme, the single grid is approximately to a square with $0.2 \mathrm{~mm}$ side length.

\subsection{Optimal distributions of groove textures under fixed eccentricity ratio}

Based on Section 4, the changes of $\mu_{\text {fbest }}$ under $\varepsilon=0.1, \varphi=0$ is illustrated in Figure 5. With increasing of generations from 1 to 30 , the $\mu_{\text {fbest }}$ shows a decreasing trend, which 


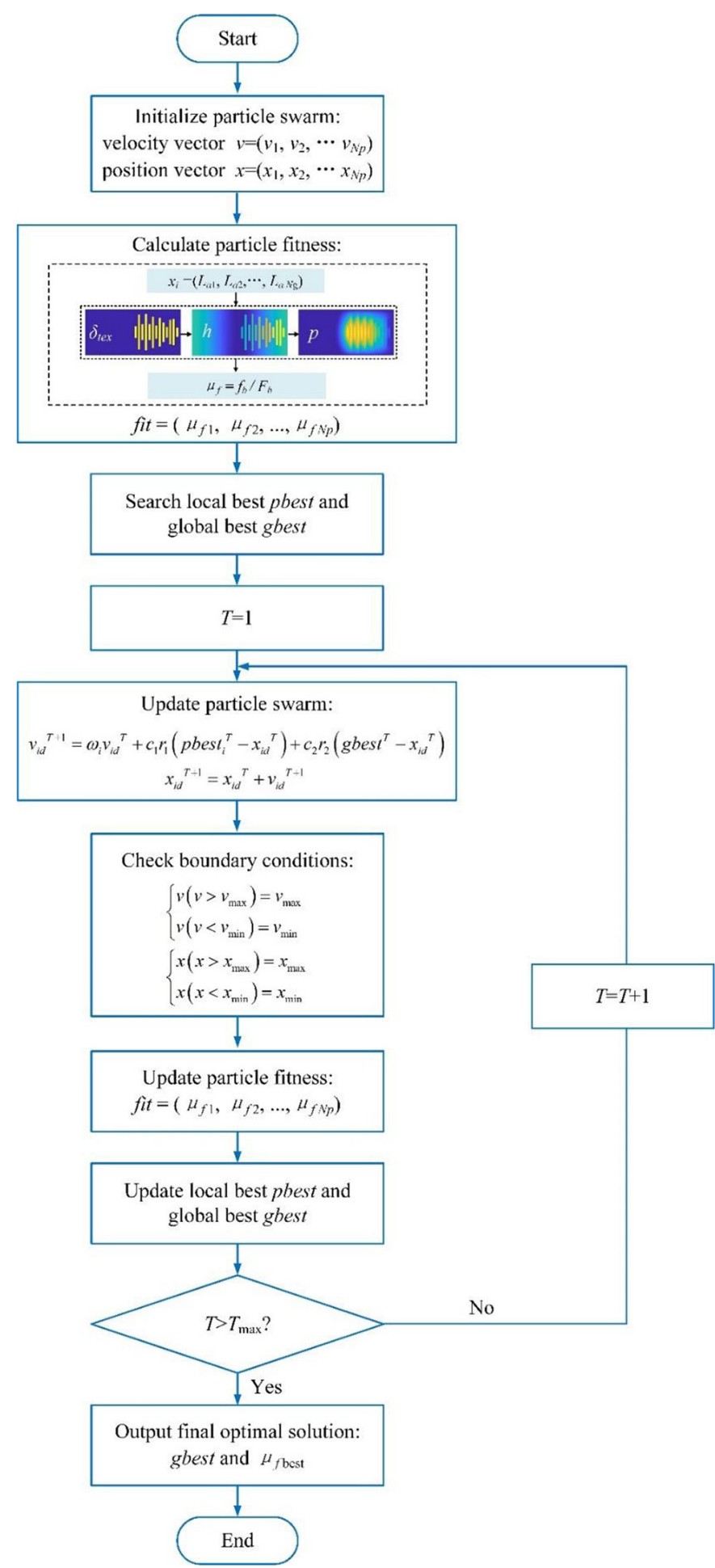

Fig. 3. Computational process of PSO algorithm.

indicates the swarm is in continuing evolution. When the generation is beyond $30, \mu_{f}$ best maintains a constant value 0.0607, which indicates the swarm has been in a stable state. The progressive changes of distributions under $\varepsilon=0.1, \varphi=0$ are shown in Figure 6 . It starts from a distribution with random groove lengths, and then gradually evolves to the final optimal distribution, whose shape is like a trapezium.

To further explain the optimal distribution of groove textures, the distribution of groove textures with an equal length $34 \mathrm{~mm}$ is given as reference, and the groove number in reference is same with it in the optimal one, as illustrated in Figure 7.

Figure 8 shows the pressure distributions of smooth, optimal textured and reference textured bearings under $\varepsilon=0.1, \varphi=0$, and Figure 9 illustrates the comparisons of load carrying capacity, friction, and friction coefficients between three bearings. It can be seen the optimal and reference textured bearings generate greater film pressures than smooth bearing due to local hydrodynamic pressures generated by grooves, which yield larger load carrying capacities. Moreover, the optimal textured bearing also generates greater pressure and larger load carrying capacity than referenced textured bearing. It can be explained that for the reference textured bearing, grooves with an equal length can destroy the pressure generations at upper and lower boundaries, that is, grooves located at upper and lower boundaries can suppress the pressure generation here. In contrast, gradually shortened grooves in optimal textured bearing reduce this pressure suppression, which yield greater pressure and load carrying capacity. Meanwhile, the frictions among three bearings are almost same. Hence, based on equation (8), the optimal textured bearing has a minimum friction coefficient, then followed by the reference textured and smooth bearings, respectively.

\subsection{Effects of eccentricity ratios on optimal distributions of groove textures}

In Section 5.1 , the eccentricity ratio $\varepsilon$ is 0.1 . In this section, the effects of eccentricity ratios $(\varepsilon=0.1-0.5)$ on optimal distributions of groove textures are discussed and shown in Figure 10. It can be seen this optimal distribution is still like a trapezium with increasing of $\varepsilon$, but it becomes slenderer. The reduction of friction coefficient between smooth and optimal textured bearings $\delta \mu_{f}$ is defined in equation (13)

$$
\delta \mu_{f}=\frac{\mu_{f \text { smooth }}-\mu_{f \text { optimal }}}{\mu_{f \text { smooth }}} \times 100 \%
$$

The friction coefficients $\mu_{f}$ and reductions $\delta \mu_{f}$ are also given. As illustrated in Figure 11, with increasing of $\varepsilon, \delta \mu_{f}$ gradually decreases from approximately $30 \%$ to $8 \%$, which indicates the reductions of friction coefficients by optimal groove textures are more significant under lower eccentricity ratios.

\subsection{Effects of rotary speeds on optimal distributions of groove textures}

In Sections 5.1 and 5.2, the rotary speed is $2000 \mathrm{rpm}$. In this section, the effects of rotary speeds on optimal 

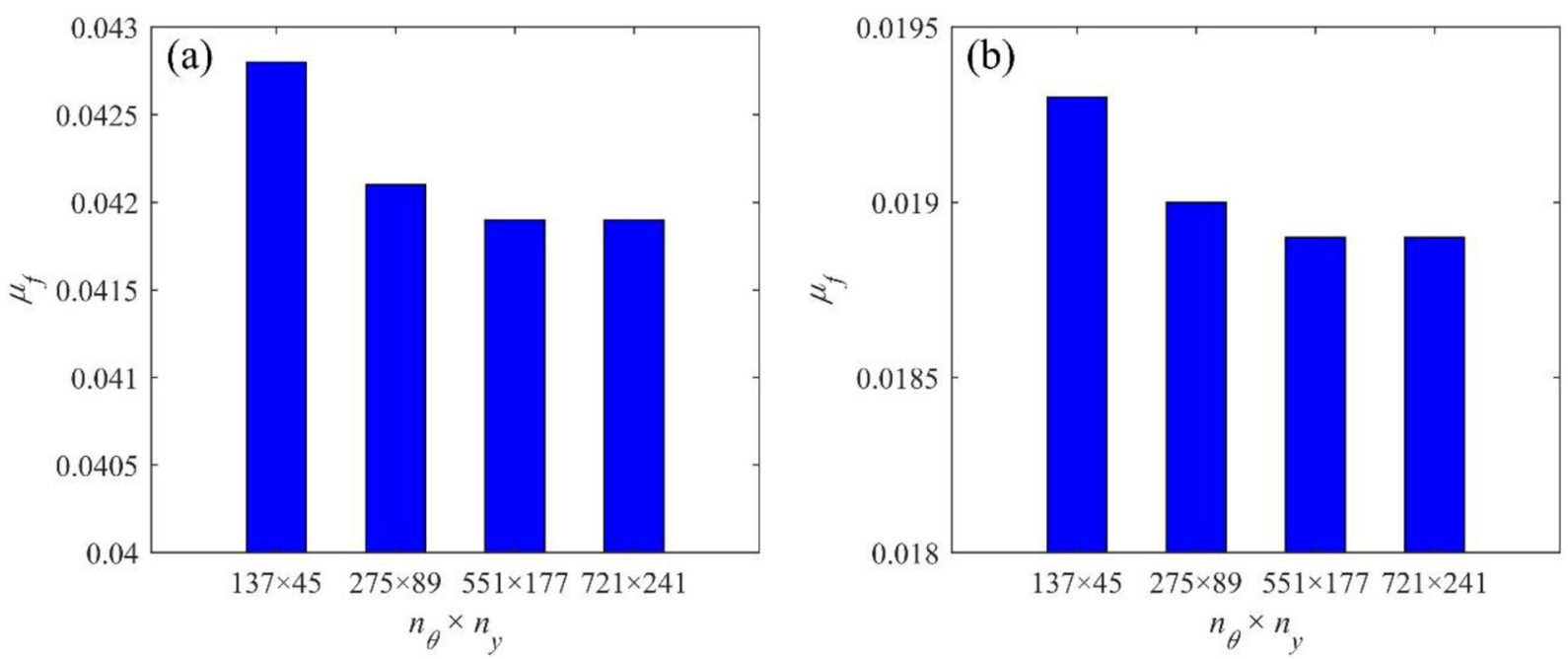

Fig. 4. Friction coefficients $\mu_{f}$ of different mesh schemes under (a) $\varepsilon=0.2$, (b) $\varepsilon=0.4$.

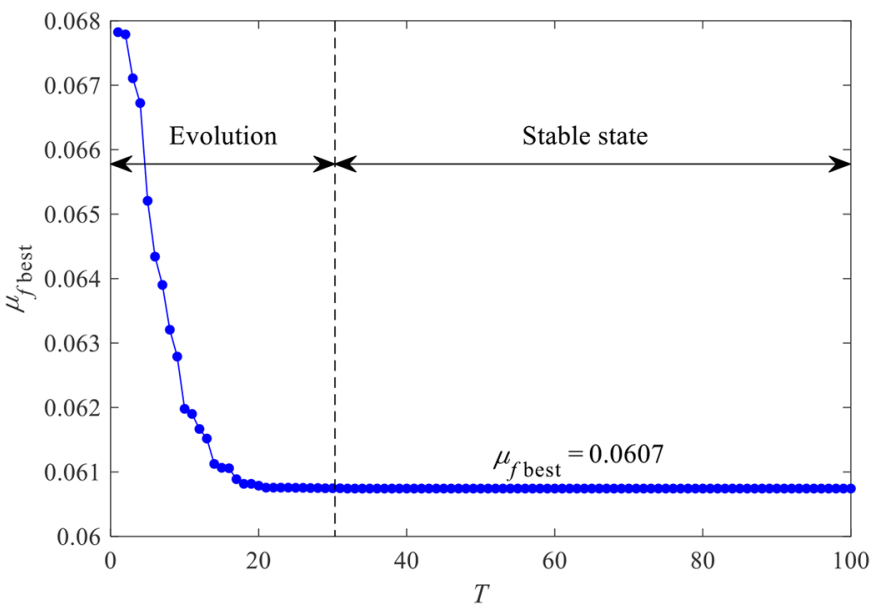

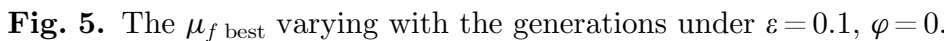
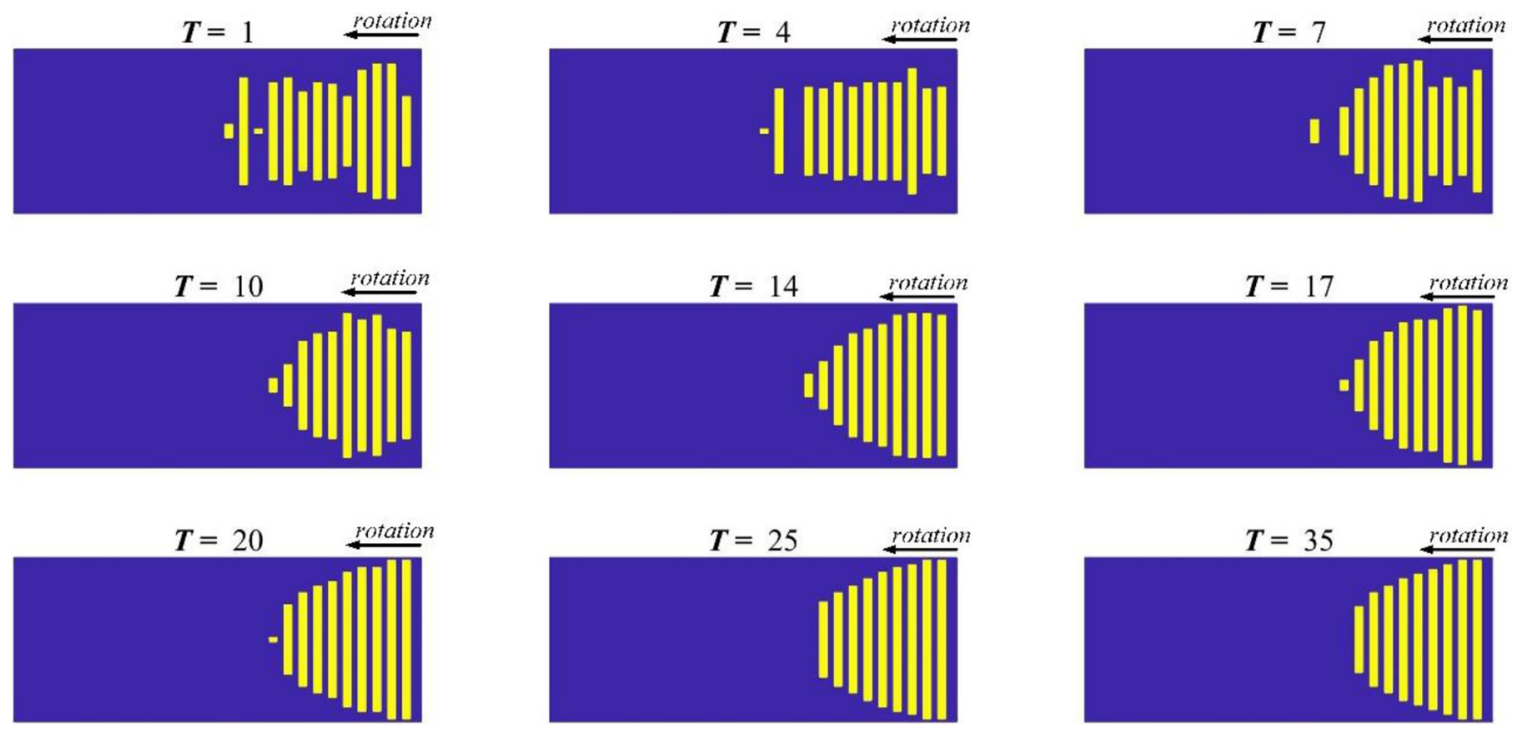

Fig. 6. The progressive changes of distributions under $\varepsilon=0.1, \varphi=0$. 

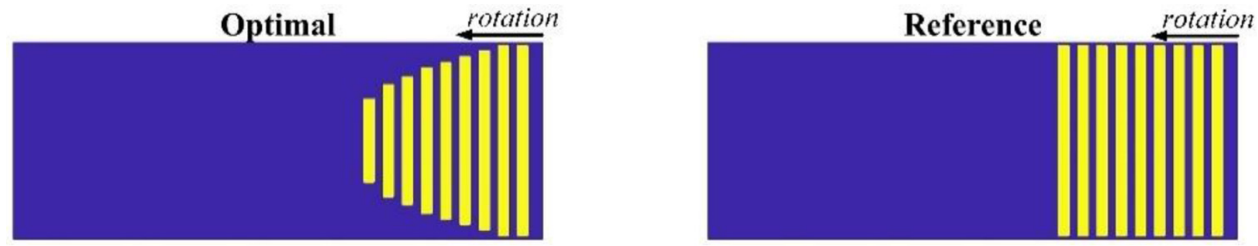

Fig. 7. The optimal and reference distributions of groove textures.
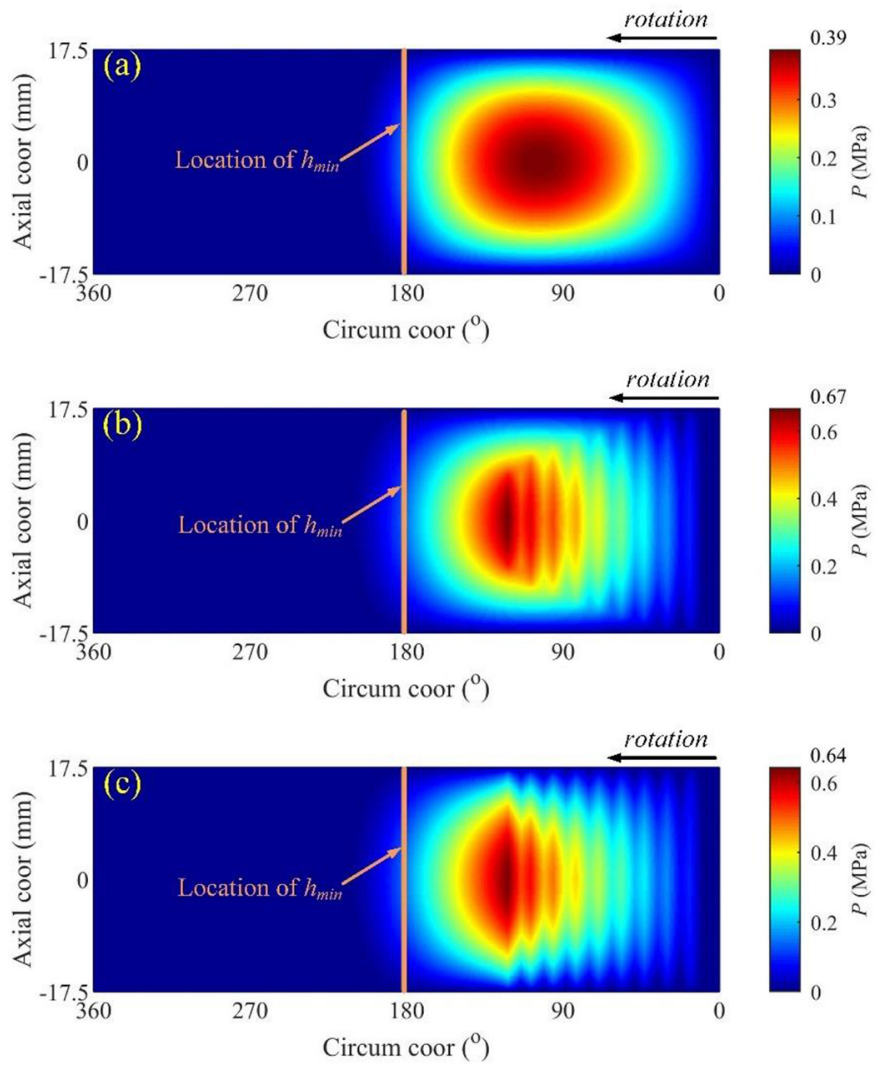

Fig. 8. Pressure distributions under $\varepsilon=0.1, \varphi=0$ of (a) smooth bearing, (b) optimal textured bearing, (c) reference textured bearing.
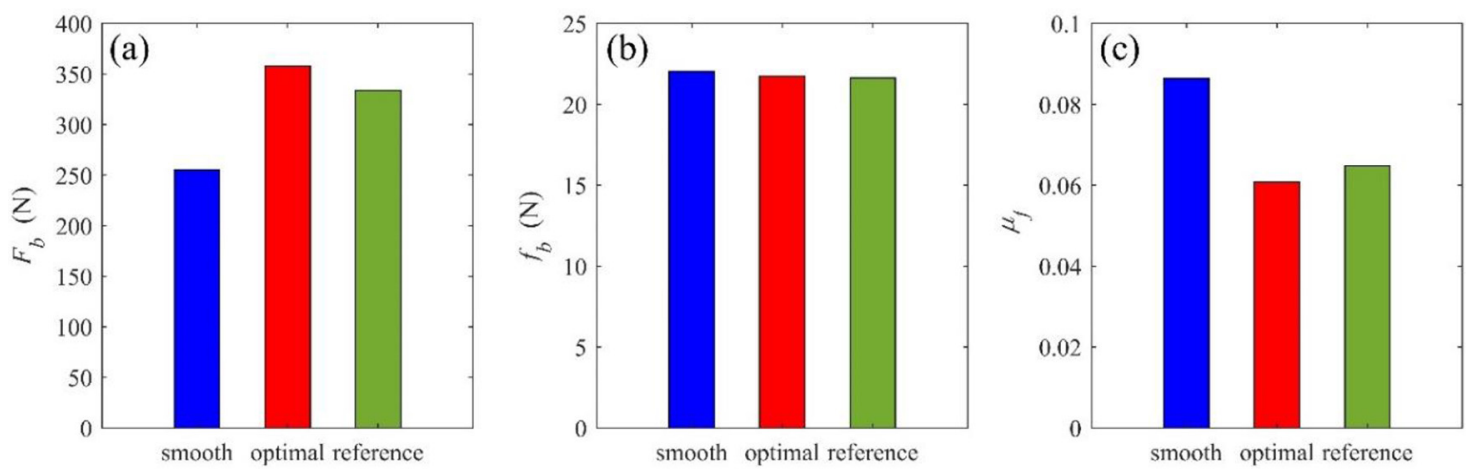

Fig. 9. Comparisons of (a) load carrying capacity, (b) friction, (c) friction coefficient, between smooth, optimal textured, and reference textured bearings under $\varepsilon=0.1, \varphi=0$. 

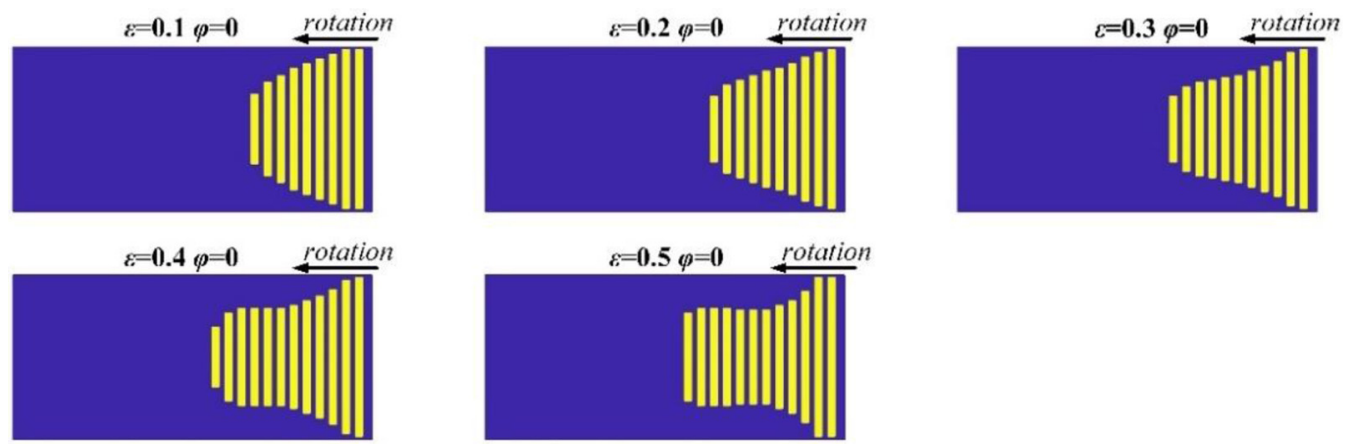

Fig. 10. The optimal groove textures under $\varepsilon=0.1-0.5, \varphi=0, N=2000 \mathrm{rpm}$.

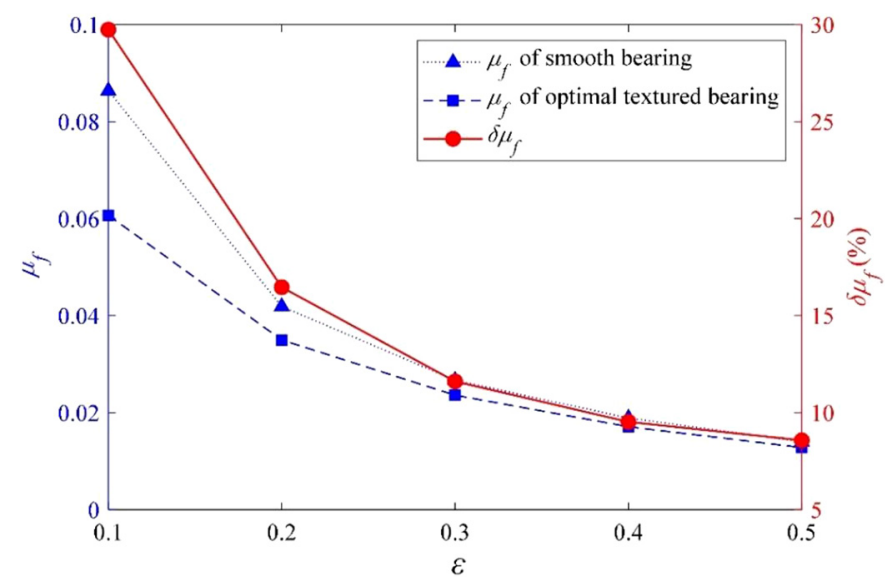

Fig. 11. $\mu_{f}$ and $\delta \mu_{f}$ under $\varepsilon=0.1-0.5, \varphi=0, N=2000 \mathrm{rpm}$.
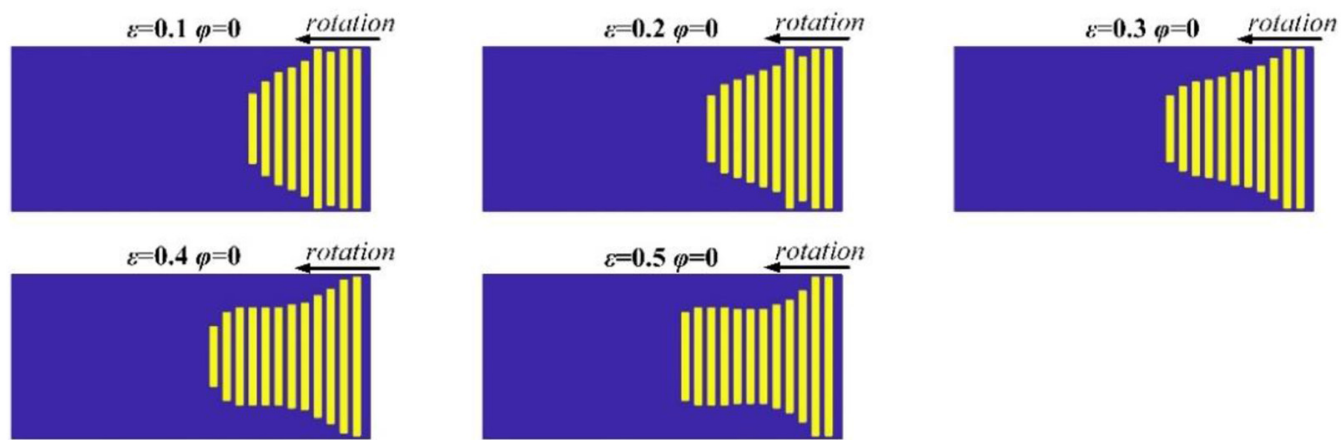

Fig. 12. The optimal groove textures under $\varepsilon=0.1-0.5, \varphi=0, N=1000 \mathrm{rpm}$.

distributions of groove textures and $\delta \mu_{f}$ are discussed. The speeds 500, 1000, 1500, 2500 and $3000 \mathrm{rpm}$ are considered. As the results of these cases are similar, only the 1000 and $3000 \mathrm{rpm}$ cases are given, as illustrated in Figures 12-15. It can be seen the results in Figures $12-15$ are similar with these in Figures 10 and 11, which indicates the optimal distributions of groove textures and trends of $\delta \mu_{f}$ are consistent under different rotary speeds.

\section{Conclusions}

This study focuses on optimizing the distributions of groove textures in a journal bearing to reduce its friction coefficient using PSO algorithm. Some conclusions are summarized below:

- For the researched journal bearing, the optimal distribution of groove textures is like a trapezium. 


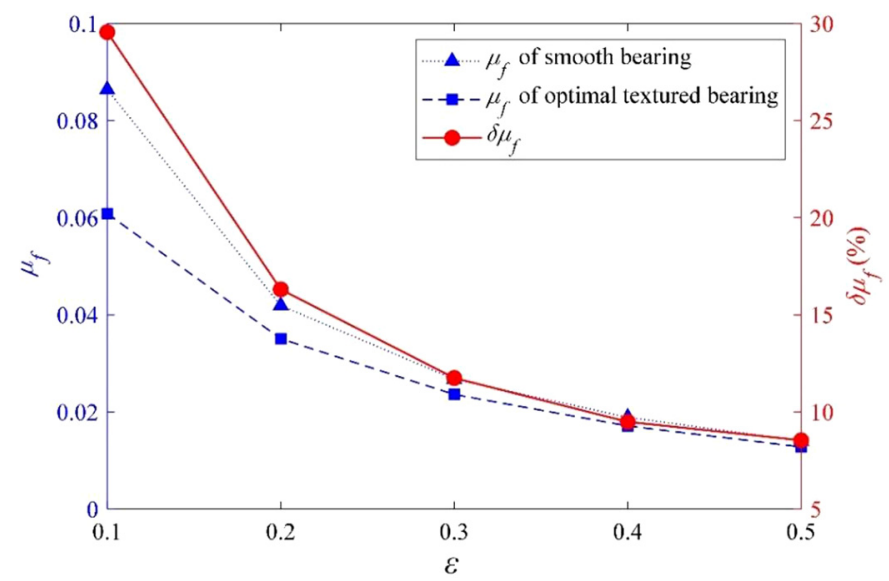

Fig. 13. $\mu_{f}$ and $\delta \mu_{f}$ under $\varepsilon=0.1-0.5, \varphi=0, N=1000 \mathrm{rpm}$.
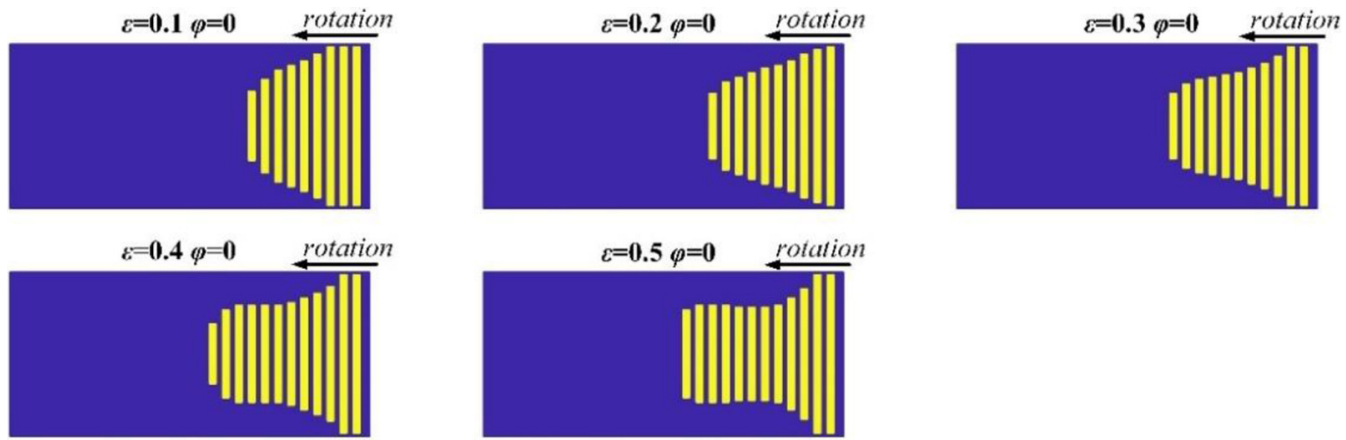

Fig. 14. The optimal groove textures under $\varepsilon=0.1-0.5, \varphi=0, N=3000 \mathrm{rpm}$.

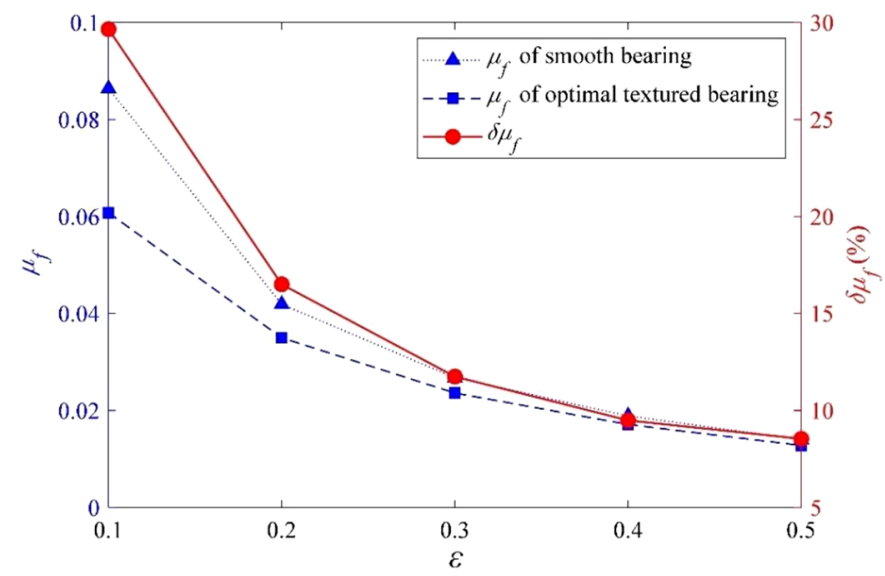

Fig. 15. $\mu_{f}$ and $\delta \mu_{f}$ under $\varepsilon=0.1-0.5, \varphi=0, N=3000 \mathrm{rpm}$.

This distribution can reduce the pressure suppression caused by grooves located at upper and lower boundaries, which yield greater film pressure and load carrying capacity. This is the main reason to reduce the friction coefficient.
- With increasing of eccentricity ratios $(\varepsilon=0.1-0.5)$, the optimal distributions of groove textures become slenderer, and the reductions of friction coefficients by optimal groove textures are more significant under lower eccentricity ratios. 
- The optimal distributions and reductions of friction coefficients are similar under different rotary speeds, which indicate the conclusions of this optimization have certain universality.

In future work, the authors will research the optimal groove textures for the journal bearing by choosing other optimization variables (such as groove depth and width) or other optimization goal (such as temperature rise), and the corresponding experiments will be performed to verify these studies.

\section{Nomenclature}

$D$

Bearing diamete

$B \quad$ Bearing width

$d_{h} \quad$ Oil hole diameter

$N \quad$ Rotary speed

$\theta_{s} \quad$ Start position of textured region

$\theta_{e} \quad$ End position of textured region

$L_{a} \quad$ Groove length in axial direction

$L_{c} \quad$ Groove width in circumferential direction

$L_{q} \quad$ Groove gap in circumferential direction

$d_{g} \quad$ Groove depth

$N_{g} \quad$ Groove numbers

$\delta_{\text {tex }} \quad$ Variation clearance caused by groove textures

$h \quad$ Film thickness

c Radial clearance

$\varepsilon \quad$ Eccentricity ratio

$\varphi \quad$ Attitude angle

$R \quad$ Journal radius

$\mu \quad$ Oil viscosity

$p \quad$ Film pressure

$\omega \quad$ Angular velocity of journal

$F_{b} \quad$ Load carrying capacity

$f_{b} \quad$ Friction

$\mu_{f} \quad$ Friction coefficient

$\omega_{s} \quad$ Overrelaxation factor

$\varepsilon_{p} \quad$ Allowable precision for the solution of pressure

$N_{p} \quad$ Particle swarm size

$v_{i d} \quad$ Particle velocity vector

$x_{i d} \quad$ Particle position vector

$\omega_{i} \quad$ Inertia weight

$c_{1}, c_{2} \quad$ Learning factors

$r_{1}, r_{2} \quad$ Random numbers between 0 and 1

pbest The local best

gbest The global best

$v_{\min } \quad$ Minimum velocity

$v_{\max } \quad$ Maximum values

$x_{\min } \quad$ Minimum groove length

$x_{\max } \quad$ Maximum groove length

$T \quad$ The index of generations

$T_{\max } \quad$ Maximum index of generations

$\mu_{f}$ best $\quad$ The minimum $\mu_{f}$ in particle swarm during each generation

$\mu_{f \text { smooth }} \quad$ Friction coefficient of smooth bearing

$\mu_{f \text { optimal }}$ Friction coefficient of optimal textured bearing

$\delta \mu_{f} \quad$ The reduction of friction coefficients between smooth and optimal textured bearings
This study is supported by National Natural Science Foundation of China (Grant No. 51809057).

\section{References}

[1] D. Gropper, L. Wang, T.J. Harvey, Hydrodynamic lubrication of textured surfaces: a review of modeling techniques and key findings, Tribology International 94, 509-529 (2016)

[2] X.H. Shen, Y.L. Shi, J.H. Zhang, Q.J. Zhang, G.C. Tao, L.J. Bai, Effect of process parameters on micro-textured surface generation in feed direction vibration assisted milling, International Journal of Mechanical Sciences 167, 105267 (2020)

[3] K. Kanda, S. Tazawa, T. Urano, S. Kobayashi, K. Adachi, The possibility of both low friction and low leakage by surface texture of mechanical seals in blood, Tribology Letters 68, 1-11 (2020)

[4] S. Ding, S. Bai, Thermoelastohydrodynamic behaviour of inclined-ellipse dimpled gas face seals, Science China Technological Sciences 60, 529-537 (2016)

[5] S. Ye, H. Tang, Y. Ren, J. Xiang, Study on the load-carrying capacity of surface textured slipper bearing of axial piston pump, Applied Mathematical Modelling 77, 554-584 (2020)

[6] D.G. Fouflias, A.G. Charitopoulos, C.I. Papadopoulos, L. Kaiktsis, Thermohydrodynamic analysis and tribological optimization of a curved pocket thrust bearing, Tribology International 110, 291-306 (2017)

[7] C. Shen, M.M. Khonsari, Tribological and Sealing Performance of Laser Pocketed Piston Rings in a Diesel Engine, Tribology Letters 64, 1-9 (2016)

[8] D. Nečas, H. Usami, T. Niimi, Y. Sawae, I. Křupka, M. Hartl, Running-in friction of hip joint replacements can be significantly reduced: the effect of surface-textured acetabular cup, Friction 8, 1137-1152 (2020)

[9] H. Kasem, H. Shriki, L. Ganon, M. Mizrahi, K. Abd-Rbo, A.J. Domb, Rubber plunger surface texturing for friction reduction in medical syringes, Friction 7, 351-358 (2018)

[10] A.B. Shinde, P.M. Pawar, Multi-objective optimization of surface textured journal bearing by Taguchi based Grey relational analysis, Tribology International 114, 349-357 (2017)

[11] S. Kango, R.K. Sharma, R.K. Pandey, Comparative analysis of textured and grooved hydrodynamic journal bearing, Proceedings of the Institution of Mechanical Engineers, Part J: Journal of Engineering Tribology 228, 82-95 (2013)

[12] B. Manser, I. Belaidi, A. Hamrani, S. Khelladi, F. Bakir, Performance of hydrodynamic journal bearing under the combined influence of textured surface and journal misalignment: a numerical survey, Comptes Rendus Mécanique 347, 141-165 (2019)

[13] R. Yu, W. Chen, P. Li, The analysis of elastohydrodynamic lubrication in the textured journal bearing, Proceedings of the Institution of Mechanical Engineers, Part J: Journal of Engineering Tribology 230, 1197-1208 (2016)

[14] Q. Lin, Q. Bao, K. Li, M.M. Khonsari, H. Zhao, An investigation into the transient behavior of journal bearing with surface texture based on fluid-structure interaction approach, Tribology International 118, 246-255 (2018)

[15] N. Tala-Ighil, M. Fillon, P. Maspeyrot, Effect of textured area on the performances of a hydrodynamic journal bearing, Tribology International 44, 211-219 (2011) 
[16] N. Tala-Ighil, M. Fillon, A numerical investigation of both thermal and texturing surface effects on the journal bearings static characteristics, Tribology International 90, 228-239 (2015)

[17] A.B. Shinde, P.M. Pawar, Effect of partial grooving on the performance of hydrodynamic journal bearing, Industrial Lubrication and Tribology 69, 574-584 (2017)

[18] X. Pang, J. Chen, S.H. Hussain, Numeric and experimental study of generalized geometrical design of a hydrodynamic journal bearing based on the general film thickness equation, Journal of Mechanical Science and Technology 26, 3149-3158 (2012)

[19] H. Zhang, M. Hafezi, G. Dong, Y. Liu, A design of coverage area for textured surface of sliding journal bearing based on genetic algorithm, Journal of Tribology 140, 061702 (2018)

[20] H. Zhang, Y. Liu, M. Hua, D.-y. Zhang, L.-g. Qin, G.-n. Dong, An optimization research on the coverage of micro-textures arranged on bearing sliders, Tribology International 128, 231-239 (2018)

[21] C. Sinanoğlu, F. Nair, M.B. Karamıs, Effects of shaft surface texture on journal bearing pressure distribution, Journal of Materials Processing Technology 168, 344-353 (2005)
[22] J. Kennedy, R. Eberhart, Particle swarm optimization, Proceedings of IEEE International Conference on Neural Networks 4, 1942-1948 (1995)

[23] R. Eberhart, J. Kennedy, A New Optimizer Using Particle Swarm Theory, Proceedings of the Sixth International Symposium on Micro Machine and Human Science (1995)

[24] N. Wang, H.-C. Huang, C.-R. Hsu, Parallel optimum design of foil bearing using particle swarm optimization method, Tribology Transactions 56, 453-460 (2013)

[25] S. Panda, S.N. Panda, P. Nanda, D. Mishra, Comparative study on optimum design of rolling element bearing, Tribology International 92, 595-604 (2015)

[26] R. Li, P.F. Du, S.M. Zhou, T.X. Zheng, Parameter optimization experiment of magnetorheological bearing, Applied Mechanics and Materials 457-458, 597-601 (2013)

[27] O. Pinkus, B. Sternlicht, Theory of hydrodynamic lubrication, McGraw-Hill, New York (1961)

[28] Y. Shi, R. Eberhart, A Modified Particle Swarm Optimizer, 1998 IEEE International Conference on Evolutionary Computation Proceedings, IEEE World Congress on Computational Intelligence (1998)

[29] Y. Shi, Particle Swarm Optimization, IEEE Connections 8-13 (2004)

Cite this article as: X. Zhang, C. Liu, B. Zhao, An optimization research on groove textures of a journal bearing using particle swarm optimization algorithm, Mechanics \& Industry 22, 1 (2021) 